\title{
Carnets
}

Revue électronique d'études françaises de l'APEF

Deuxième série - 14 | 2018

Études de génétique théâtrale et littéraire

\section{Critique de processus de création : une approche théorique possible}

\section{Cecilia Almeida Salles}

\section{(2) OpenEdition}

\section{Journals}

\section{Édition électronique}

URL : http://journals.openedition.org/carnets/8913

DOI : 10.4000/carnets.8913

ISSN : 1646-7698

Éditeur

APEF

\section{Référence électronique}

Cecilia Almeida Salles, "Critique de processus de création : une approche théorique possible », Carnets [En ligne], Deuxième série - 14 | 2018, mis en ligne le 30 novembre 2018, consulté le 02 mai 2019. URL : http://journals.openedition.org/carnets/8913 ; DOI : 10.4000/carnets.8913

Ce document a été généré automatiquement le 2 mai 2019.

\section{(c) (i) (8)}

Carnets est mis à disposition selon les termes de la licence Creative Commons - Atribution - Pas d'utilisation commerciale 4.0 International. 


\title{
Critique de processus de création: une approche théorique possible
}

\author{
Cecilia Almeida Salles
}

1 La recherche sur les processus de création, développée à l'Université Pontificale Catholique de São Paulo / Brésil (PUC/SP) depuis 1990, surgit face à la nécessité de traiter des documents d'une grande diversité de manifestations artistiques comme la littérature, le théâtre, le cinéma, la danse, etc. Une telle documentation offre au chercheur des archives de la création sous forme de journaux, cahiers d'artistes, notes, registres audiovisuels, etc., qui viabilisent la compréhension des principes qui guident les processus créatifs des artistes étudiés. Les registres analogiques et/ou numériques du parcours sont faits dans le langage le plus accessible à l'artiste dans le moment, qu'il soit écrit, oral ou visuel. L'approche de telles archives, comme indices du développement de la pensée en processus, ouvre la possibilité que soient faites des études dans d'autres domaines comme la traduction, la philosophie, la science, etc.

2 Il s'agit d'une recherche qui est apparue à partir de l'étude de la littérature brésilienne contemporaine, en dialogue avec les études de critique génétique. Si le but directeur de telles études, à ses débuts en France à l'Institut des Textes et Manuscrits Modernes (ITEM/CNRS/Paris), était la compréhension du processus de production d'une œuvre littéraire et si leur objet d'étude était les registres du parcours de l'écrivain, elles pourraient rompre la barrière de la littérature et étendre leurs limites au-delà de la parole. Le processus et les registres sont indépendants de la matérialité dans laquelle l'œuvre se manifeste et indépendants, aussi, des langages dans lesquels ces empreintes se présentent. Ainsi, il était possible de connaître certains procédés de la création, dans n'importe quelle manifestation artistique, à partir de la grande diversité de pistes laissées par les artistes.

3 Il est intéressant d'observer que la critique génétique passe par des ajustements au fur et à mesure qu'elle se développe. Au nom de son expansion, elle subit plusieurs ajustements méthodologiques, terminologiques et théoriques. Comme exemple, nous avons la difficulté d'utiliser le terme manuscrit avec d'autres manifestations artistiques. J'ai cherché un autre mot : document de processus semble remplir cette tâche, en soulignant 
le rôle joué par les registres - le besoin de retenir certaines idées ou actions - et non la matérialité. Ainsi, on peut parler de documents sous forme de notes, de journaux, aussi bien que de répétitions dans les arts de la performance, de registres audiovisuels, etc., incluant tout le potentiel offert par les médias numériques. Dans cette optique, les nouvelles technologies, au lieu d'indiquer la fin de ces documents, contribuent à l'augmentation de leur diversité et à leur expansion constante.

Les registres analogiques et/ou numériques du parcours sont faits dans le langage le plus accessible à l'artiste au moment où il ressent le besoin, qu'il soit écrit, oral ou visuel. De ce point de vue, la pensée en création se produit en un mouvement continu de traduction de langages comme, par exemple, des mots et des images qui se transforment en action dans le corps d'un acteur.

5 De façon assez générale, ces registres remplissent deux fonctions: stockage et expérimentation. L'artiste rencontre les plus divers moyens de stocker des informations qui jouent le rôle d'auxiliaires dans le parcours de concrétisation de l'œuvre et qui nourrissent l'artiste et l'œuvre en création. Il y a d'autres documents qui enregistrent l'expérimentation, laissant transparaître la nature inductive de la création. Dans ce moment de concrétisation de l'œuvre, des hypothèses de natures diverses sont émises et peu à peu testées. Ce sont des possibilités d'œuvres, rencontrées dans des répétitions ou des versions différentes du texte théâtral, par exemple.

6 Stockage et expérimentation sont communs à la grande majorité des artistes; les singularités surgissent en ce qui est préservé, tel que c'est enregistré et, dans le cadre de l'expérimentation, dans les critères des choix de chaque artiste. Les différents documents de processus, ceux auxquels le chercheur a accès, génèrent différents types d'information qui désignent des aspects ou des moments différents des processus de création.

7 Il s'agit d'une recherche qui offre la possibilité de faire une investigation à partir de la matérialité des processus, offrant un concept de création en opposition à la vue d'un fascinant et inexplicable déclic, sans histoire et sans couches de travail.

\section{Quelques considérations méthodologiques}

8 Ce sont des études critiques des parcours de production développées à partir d'une approche phénoménologique : les propres documents servent de guide contrôleur pour les interprétations. La question qui nous guide est « qu'est-ce que ce matériel nous offre sur le processus créatif de l'artiste étudié ?»

9 L'observation attentive des documents est développée dans une perspective relationnelle : le critique établit des relations entre les différentes données pour refaire et comprendre le réseau de la pensée en création. C'est dans l'établissement de relations entre les actions et les décisions de l'artiste que l'on perçoit certains des principes directeurs de ce processus. L'observation relationnelle conduit à la formulation d'hypothèses sur le mode dont le processus de création se développe. La méthodologie de ces recherches est donc basée sur ce que Morin (2000: 23) décrit comme «l'art de transformer des détails apparemment insignifiants en indices qui permettent de reconstruire toute une histoire ».

10 Le chercheur quête des explications pour le processus créatif. Par conséquent, de simples descriptions ou récits s'avèrent insuffisants. De la complexité des informations, on retire 
le système engendré par le processus. Un accompagnement critico-interprétatif des registres est fait.

11 La méthodologie est aussi, depuis un moment, en processus d'expansion: plusieurs chercheurs, spécialement dans le cas du théâtre et de la danse, commencent à suivre des processus générant d'autres documents sous la forme de notes, de photographies, d'enregistrements audiovisuels, etc.

12 Il est intéressant d'observer que de nombreux groupes de théâtre ou de danse invitent des cinéastes et/ou photographes pour enregistrer leurs parcours (ateliers, improvisations et répétitions), générant du matériel de recherche qu'alimentent les propres membres des groupes. En même temps, ils offrent une documentation pour les chercheurs intéressés par des processus de création.

13 Ces registres photographiques et audiovisuels de processus de création relèvent ainsi, ces derniers temps, les défis de documenter et d'archiver des processus créatifs dans le théâtre, la danse, la musique et la performance. Ce qui est observé, c'est que les archives de création sont en pleine expansion, spécialement dans l'interaction avec les expérimentations artistiques contemporaines, comme nous le verrons.

14 Le chemin choisi pour la réflexion proposée dans cet article était de partir du plus général pour arriver au plus spécifique.

\section{Proposition théorique}

Depuis le début de l'histoire du développement de la critique génétique telle qu'elle est développée par les Français dans l'ITEM, les recherches ont toujours montré une forte tendance à la quête de la singularité des processus créatifs spécifiques.

Quelques chercheurs avancent aujourd'hui vers une généralisation du processus de création, menant à la cartographie de principes qui guident une théorie possible de la création, qui soutient ce que j'appelle critique de processus créatifs ou simplement « critique de processus ». Comme l'affirme Colapietro $(2014)^{1}$ : « penser, c'est généraliser pour jeter quelque lumière sur le spécifique ».

17 C'est dans ce contexte que je présente les recherches du Groupe d'Études de Processus de Création, développées dans le Programme de $3^{\text {e }}$ cycle de Communication et Sémiotique de l'Université Pontificale Catholique de São Paulo (PUC/SP).

$18 \mathrm{Au}$ fur et à mesure qu'une théorie possible de la création est configurée, il y a une inversion de perspective : les études sur les histoires d'œuvres spécifiques se mettent au service de quelque chose de plus ample qui est la théorie du processus créateur. Le parcours de la création se présente comme un réseau d'actions qui laissent transparaître des récurrences significatives qui rendent possible la rencontre d'aspects généraux sur le faire créatif, qui permettent de jeter quelque lumière sur le spécifique avec plus d'acuité. Ce ne seraient pas des modèles d'analyse qui, normalement, fonctionnent plutôt comme des moules théoriques qui rejettent ce qui ne leur convient pas. Ce sont des instruments théoriques qui permettent l'activation de la complexité du processus. Il s'agit, donc, d'une théorie possible de la création qui a eu comme point de départ des études singulières de documents et qui, en même temps, s'alimente de ces mêmes recherches. Il y a des questions générales dans les processus, mais qu'est-ce qui fait, par exemple, que le 
théâtre soit du théâtre ou qu'est-ce qui caractérise le processus de création d'un acteur ou d'un metteur en scène spécifique?

On ouvre la possibilité de développer des recherches comparées, tant en ce qui concerne différents auteurs d'un même moyen d'expression qu'en ce qui concerne des processus de créateurs de différents domaines.

Une telle théorisation a commencé à offrir une approche procédurale, ajoutant au regard rétrospectif de la critique génétique, une dimension prospective d'une critique de processus créatifs. Cela a permis de discuter des questions de procédures qui dépassent les limites des histoires de comment les œuvres ont été construites. Il convient de souligner l'importance de proposer une théorie critique procédurale face aux expérimentations et aux interrogations artistiques contemporaines qui impliquent, entre autres choses, des explorations d'archives et des différentes relations entre processus et œuvre, comme nous en discuterons plus en avant, à partir d'une œuvre de Jérôme Bel.

Si ce que nous recherchons est la meilleure compréhension de la complexité qu'implique le processus créatif, nous ne pouvons pas nous servir de concepts théoriques isolés. Je crois que la discussion sur la création doit aussi se faire en réseau, à l'aide d'un corps théorique avec des concepts organiquement interconnectés.

22 Je présente, ainsi, le concept de "création comme réseau en construction ", de base sémiotique (Peirce et Colapietro), en dialogue avec le concept de réseau (Musso) et avec des penseurs de la complexité comme Morin.

23 D'un côté, il s'agit d'une proposition ouverte à d'autres théories, nécessaires aux spécificités des artistes ou groupes étudiés et significatives pour les investigations de chaque chercheur ; en même temps, chaque processus demande une interaction avec des théories de cette manifestation spécifique, en d'autres termes, les instruments théoriques de nature générale, que nous présenterons ensuite, doivent dialoguer avec les théories du théâtre, de la danse, etc.

24 Cette conception théorique est soutenue par le concept de réseau de Pierre Musso (2004). Le terme " en construction ", de son côté, était la forme trouvée pour relier au concept de réseau celui de sémiose (en termes peirciens) qui propose la lecture de la création comme processus continu avec des tendances ou des propositions. ${ }^{2}$

Il y a d'autres questions importantes, générées par la perspective peircienne, qui donnent corps à cette conceptualisation de création. Il s'agit d'un parcours d'action (construction / conflit) sensible et intellectuel ${ }^{3}$, souffrant des interventions du conscient et de l'inconscient. Il s'éloigne, ainsi, de la dichotomie intellectuelle ou sensible qui régit encore les discussions de nombreux penseurs de la création, traitant, par exemple, le processus de la science comme objectif et intellectuel et celui de l'art comme subjectif et sensible. Ce qui est proposé ici, c'est que tous les processus coexistent avec ces trois aspects. On pourrait dire que les différences sont d'un autre ordre, comme la nature de la quête.

26 La création comme réseau peut être décrite comme un processus continu d'interconnexions, avec des tendances vagues, générant des nœuds d'interaction dont la variabilité obéit à des principes directeurs. Ce processus continu, sans point initial ni final, est un mouvement faillible, soutenu par la logique de l'incertitude, englobant l'intervention du hasard et ouvrant un espace pour l'introduction d'idées nouvelles. Les interconnexions nous placent dans le champ relationnel: toute action est liée à d'autres de même importance, étant ainsi un parcours non linéaire et sans hiérarchies. La 
construction d'un réseau, c'est-à-dire sa transition d'un réseau simple à un autre plus complexe est consubstantielle à sa définition. Les interconnexions génèrent les nœuds du réseau, éléments d'interaction reliés entre eux, qui se manifestent comme les axes directeurs de nos recherches.

Les tendances sont des directions vagues qui guident le processus de construction des objets, dans l'ambiance d'incertitude et d'imprécision. Elles génèrent un travail à la recherche de quelque chose qui est à découvrir. Les tendances peuvent être observées sous deux perspectives: la constitution de projets poétiques et les pratiques communicatives. Certaines de ces questions seront reprises, quand nous parlerons des spécificités du théâtre.

La «récompense matérielle », c'est-à-dire le parcours de construction du réseau, est insérée dans l'espace et le temps de la création, qui affectent inévitablement l'artiste. Le terme est emprunté à Kandinsky (1990) qui décrivait le processus de construction d'œuvres comme la recherche de la récompense matérielle pour le pouvoir inventif et la sensibilité de l'artiste.

Tout au long de ce processus, sont, petit à petit, stipulées des restrictions ou des délimitations de natures diverses qui rendent possible la construction de l'œuvre. Le développement du processus mène à la prise de décisions qui favorise la formation de lignes de force, donnant une consistance aux objets en construction.

31 Le concept de tendance est emprunté à la définition de sémiose (mouvement du signe) en termes peirciens. Selon l'auteur tout processus signique porte le concept de but, d'objectif, et implique une lutte pour l'obtenir.

L'utilisation du terme "poétique » pour désigner la nature du projet, qui régit les processus de création, implique des aspects éthiques et esthétiques (Peirce) des recherches. Nous pouvons parler, donc, de projet théâtral, littéraire, etc. L'important est de porter avec les termes le mode d'action des tendances, c'est-à-dire un «désir opératoire » (Peirce, CP 1. 205) ${ }^{4}$

Dans ce contexte de tendances vagues, le projet est, donc, constitué par des principes directeurs présents dans les pratiques créatrices, liés à la production d'une œuvre spécifique et qui relient l'œuvre de l'artiste en question comme un tout. Ce sont les théories implicites dans le faire, relatives à la singularité de l'artiste. Ce sont des valeurs, des formes de représenter le monde, des goûts et des croyances qui régissent son mode d'action. La recherche, comme processus continu, est toujours incomplète. Le projet luimême, qui dirige d'une certaine façon la production des œuvres, change au cours du temps.

Du point de vue des pratiques communicatives, le processus de création se révèle comme une tendance vers les autres, dans la mesure où il est inséré dans les réseaux culturels complexes. L'aspect communicatif du processus implique des sujets en tant que communauté, établissant une grande diversité de dialogues de nature inter et intrapersonnelle.

Nous utilisons le concept de « sujet en communauté » développé par Colapietro (1998 et 2003). Il présente le sujet, d'un point de vue sémiotique, comme un être historique et concret, culturellement surdéterminé, inséré dans un réseau de relations, et focalise, sous une autre perspective, l'inévitable insertion dans la chaleur et l'imprinting culturel (Morin, 1998). Pour Colapietro (1989), nous sommes toujours au milieu d'autres personnes et d'autres significations. «Sujet » est une source non primordialement libre de pensée et 
d'action mais un être profondément incrusté dans son temps et son espace, au point d'être assez, mais non complétement, limité dans sa cognition et sa conduite.

Colapietro (1989) souligne que le sujet n'est pas une sphère privée. Il est distinguable, cependant il n'est pas séparable des autres car son identité est constituée par les relations avec les autres; il n'est pas seulement un membre possible d'une communauté, mais la personne comme sujet a la forme même d'une communauté.

En discutant, de façon plus spécifique, de la créativité, Colapietro (2003) parle de l'impossibilité d'identifier son local avec l'imagination, spécialement quand l'imagination est conçue comme un pouvoir inhérent à la psyché individuelle; en d'autres termes, le local de la créativité n'est pas l'imagination d'un individu. Il souligne, donc, qu'il est impératif de parler de locaux de la créativité, ceux où les pratiques interagissent. Dans le changement de la perspective du sujet en lui-même pour l'explication du sujet du point de vue des pratiques entrelacées, le locus de la créativité est pluralisé et historicisé.

38 À partir de ces réflexions en interaction avec le concept de réseau, je propose une conceptualisation d'auctorialité, dans le contexte des interactions entre le sujet et les autres. L'auctorialité s'établit dans les relations. Il s'agit d'un concept d'auctorialité en réseau.

39 C'est une auctorialité distinguable, mais non séparable des dialogues avec l'autre; il ne s'agit pas d'une auctorialité fermée en un sujet. Cependant, il y a encore de l'espace pour la distinction, autrement dit, la multiplicité d'interactions n'implique pas un effacement absolu du sujet.

40 De ce point de vue, les agents en création sont observés au sein de la multiplicité des interactions et des dialogues: des sujets constitués et situés qui trouvent des modes de manifestation dans des failles que leurs filtres médiateurs conquièrent.

41 Les archives de la création se présentent, souvent, comme un espace de stockage de cette multiplicité d'interactions, et comme l'impossibilité conséquente de parler de processus individuels ou de sujets isolés. En d'autres termes, la matérialisation de ces interactions est préservée dans les documents de processus, faisant que le chercheur cohabite avec l' « effervescence » des sujets : ses choix, préférences, récurrences du regard, références, etc. C'est-à-dire, ils laissent des marques de leur subjectivité.

À partir de la présentation de ces instruments théoriques de nature générale, je passe à la discussion de certains de ces aspects pour arriver à certaines singularités des processus en groupe, plus spécifiquement du théâtre.

43 Je recours à Eisenstein (1987: 101) pour donner une plus grande complexité à cette discussion. Le cinéaste fait une analogie de la production cinématographique avec la construction de ponts et avec le travail du musicien d'orchestre. Au milieu des collaborations, commandements et hiérarchie, en définissant les processus en équipe, il souligne qu'il s'agit de l'« entrelacement d'actes individuels avec l'action générale ». Cela a des déploiements intéressants pour réfléchir sur le mode d'action du collectif : ce sont des individus qui permettent les productions en équipes visant une action générale ou un projet commun. Comme on peut le voir, on ne peut pas penser en groupe, sans parler du processus de chaque sujet. J'ajoute encore des sujets en communauté.

44 Les processus de création en équipe gagnent, donc, une plus grande complexité quand on pense à chacun des membres du groupe, expérimentant ses propres réseaux, en intense interaction avec ceux des autres, construisant un projet commun, dans le cadre des principes qui régissent le groupe dont il fait partie. 

que l'on cherche, autrement dit, différentes manières d'organisation du groupe engendrent de nouvelles possibilités artistiques. En ce qui concerne les modes de travail, je parle des plus hiérarchiques ou de toute la diversité de formes nommées collaboratives. déploiements qui apparaissent avec récurrence dans les rapports et les accompagnements de processus, comme : nature du projet commun, types de relations, modes de direction, appartenance à la communauté, conflits, affinités, adhésion, ressources financières, contexte culturel et politique propices et/ou adverses. groupes qui ont fait partie de sa recherche ; cependant, on peut observer la même chose dans d'autres contextes de création, d'autres moments historiques et d'autres natures de quêtes, comme dans le cas du théâtre. Il dit que le leader doit être quelqu'un capable d'amalgamer des efforts et des concepts pour la construction d'un projet commun. groupe et des interactions de la construction d'un projet théâtral et des pratiques communicatives, mentionnées antérieurement. processus, de lectures de journaux, de lettres et du dialogue avec les études de Domenico de Masi (2007) sur des groupes créatifs européens de 1850 à 1950. du théâtre, il est important de parler de certaines questions qui impliquent la formation des groupes, c'est-à-dire le moment où les sujets en communauté se réunissent et deviennent groupe.

\section{Formation du groupe} partir de différentes personnes impliquées dans ce parcours de nature collective, offrant différentes portes d'entrée pour la compréhension de tels processus : metteur en scène, acteur, dramaturge, éclairagiste, etc. Dans l'interrelation de ces données nous pouvons comprendre la complexité du groupe. plus stables, d'autres avec certains membres fixes et d'autres non. Il y a des entrés et des sorties, des personnes participant à plus d'un groupe, etc. Ce sont des partenariats normalement établis par affinités esthétiques et éthiques et en accord avec les projets qui sont développés.

On mentionne les conflits pour maintenir des compagnies de théâtre pendant longtemps, car cela implique les recherches artistiques de chaque membre, les différents projets de vie, canalisés vers un idéal commun qui est repensé de temps en temps, générant des conflits et des allées et venues de ses membres.

Un autre aspect observé, c'est que différents modes de travail sont étroitement liés au théatre que l'on cherche, autrement dit, différentes manières d'organisation du groupe Les questions qui impliquent la formation de l'équipe sont en relation avec certains

Ce sont ces difficultés de construire un projet du groupe que je discute désormais du point de vue du concept de création en tant que réseau. Je crois que penser l'interrelation 
des deux dimensions des tendances des processus, déjà mentionnées, peut être un chemin fertile pour comprendre certaines des spécificités des processus théâtraux.

Dans ce contexte, je réfléchis sur la construction des projets communs et le processus de communication des projets en construction.

\section{Construction du projet commun au groupe entre les pratiques communicatives}

Le projet se constitue petit à petit dans les relations avec les membres du groupe, donc, au milieu de pratiques communicatives.

L'acte d'adhésion à un projet commun nous mène à un questionnement de Morin (2010), dans le contexte académique. Il affirme que les relations entre scientifiques sont de nature amicale et hostile, de collaboration et de compétition, régies par le jeu de vérification. Cela nous conduit à un intéressant espace de réflexion sur d'autres processus : quelles seraient les règles du jeu des autres activités en groupe comme le théâtre?

Pour réfléchir sur ces règles, je souligne à nouveau l'importance de comprendre les implications des prises de décision, des critères, basés sur les principes directionnels des membres de l'équipe et, par conséquent, des commandements. Dans ce contexte, les critères sont les règles de vérification internes à la construction de ce projet qui est évalué en permanence.

59 La difficulté de coexister avec l'incertitude dans la collectivité est souvent explicitée. Le projet qui dirige les actions du groupe est, comme nous l'avons vu, immergé dans cet univers de vague et d'imprécision. Le développement $d u$ processus mène progressivement à certaines prises de décisions qui permettent la formation de lignes de force. Celles-ci, à leur tour, se mettent à soutenir les œuvres en construction et balisent, d'une certaine façon, les évaluations. Des prises de décision impliquent des critères qui ne sont rien d'autre que ce dont sont faits les projets artistiques: des fils conducteurs qui guident l'action artistique.

Antônio Araújo $(2011: 137)^{5}$, metteur en scène de théâtre, en abordant les prises de décision dans les processus collaboratifs, parle de concepts structurants qui seraient ces critères nécessaires dans l'affrontement d'impasses: les responsables de chaque secteur (metteur en scène, dramaturge, éclairagiste, acteurs, etc.) « auraient le droit à la parole finale concernant cet aspect de la création ». "Concept structurant » ou " parole finale » sont des indices de critères de prises de décision qui rendent le projet réalisable ou non. Et ainsi sont délimités des principes directeurs en commun, diminuant le vague.

Dans le témoignage sur le processus du théâtre du Vertige, il est clair que ce projet théâtral commun est instauré dans la collectivité, dans le contexte d'un certain mode de travail, collaboratif, c'est-à-dire des fonctions maintenues mais sans hiérarchie avec des contaminations mutuelles entre les artistes. Il constate que son imagination est comme provoquée par des éléments hors de moi et le corps de l'acteur, dans ce sens, fonctionne comme une espèce de déclencheur. L'expérience et la présence de l'autre sont, dans ce sens, des enzymes pour mes mécanismes créatifs. (Araújo, 2011). Nous comprenons, ainsi, les prises de décision dans l'ambiance collaborative du point de vue de ce metteur en scène. 

un leader qui convoque pour la réalisation d'un projet par lui idéalisé ou proposé. Cependant, cette équipe est formée par des sujets avec leurs propres désirs et leurs propres quêtes, comme nous l'avons vu. considérés comme responsables de bons résultats, apporte d'autres aspects intéressants pour la discussion de la construction du projet commun. Il parle d'affinité culturelle et d'adhésion en termes de concentration, au fil du temps, d'énergie au projet commun du groupe.

Réfléchissant, encore, à la construction du projet artistique dans le cadre de la collectivité, j'apporte une grande diversité de documents de processus associés à la singularité des groupes, comme, par exemple, les registres audiovisuels du parcours de création dans le théâtre, faits par des membres du propre groupe ou sollicités à un cinéaste, comme nous l'avons vu. Ils semblent être des moyens de ne pas perdre le potentiel des interactions si multiples, qui arrivent lors des répétitions, dans des ateliers, etc.

Il est clair que cette amplification des documents de processus, dans le cadre des groupes, est associée aux nouvelles technologies, avec des appareils photo et/ou des téléphones portables, mais spécialement aux propositions du mode de travail: le processus collaboratif, par exemple, mise sur la force des propositions de tous les membres du groupe et sur la nécessité de retourner à ces multiples interactions pour faire des choix dans le parcours de la construction de projets artistiques, instaurés dans la collectivité.

En poursuivant la proposition de discuter des spécificités des réseaux des processus théâtraux, je passe au deuxième aspect observé : la communication du projet artistique.

\section{Communication du projet artistique}

Devant les difficultés de la communication entre les membres du groupe, le chorégraphe et danseur Alwin Nikolais (apud Murray, 1992: 133) dit que «chorégraphier pour une autre personne est comme remettre à quelqu'un une poignée de sable. Beaucoup de choses se perdent. Mais on apprend à se contenter d'un pourcentage raisonnable ».

L'accompagnement des processus de création souligne, dans ce contexte spécifique, que le sensible accompagne tout le parcours: collectes sensibles, recherche d'un effet esthétique, émotions face aux rencontres, doutes et conflits, etc.

Pour cette discussion il est important de reprendre aussi le fait que la création est un parcours de recherche de la construction de projets, dans le cadre du vague des tendances et de l'incertitude des chemins pris. Ainsi une grande partie de ce que l'on désire de l'œuvre est ressenti et ne gagne des contours plus nets que tout au long du parcours. Dans les processus de théatre, cette question devient plus complexe, dans la mesure où ce qui semble être ce que l'on veut de l'œuvre doit être transmis aux autres membres du groupe.

Quant au mode d'exprimer cette quête sensible, on observe, dans les notes de journaux d'acteurs, par exemple, la mention de l'utilisation, de la part du metteur en scène, de figures de langage comme des métaphores et des comparaisons, aussi bien que d'adjectifs, avec toute la charge de subjectivité qu'ils portent. On pose ainsi la question du langage qui, comme nous le savons, échappe à la précision et a la fonction de clarifier la 
proposition de la quête, dans un moment encore nébuleux. Cet état d'incertitude génère une expérimentation sous la forme de répétitions, d'ateliers, etc. Ainsi, lors des différentes tentatives, de matérialité diverse, des choix apparaissent, conduisant à certains critères dans le cadre de ce projet, comme il a déjà été mentionné. Le projet luimême est mieux compris quand certains choix commencent à gagner une plus grande consistance.

71 En ce qui concerne les pratiques communicatives des agents en création, on fait des registres de dialogues entre les membres de l'équipe, c'est-à-dire des instruments de passage ou des médiateurs. Cependant, il y a encore une grande diversité de ces registres concernant les spécificités du groupe et/ou des sujets impliqués.

Dans de nombreux cas, ce qui paraît être en jeu est la tentative et la nécessité de passer aux autres les principes directionnels de ce projet en construction. Ces archives ont une grande importance pour les chercheurs intéressés par le processus de création car elles préservent des indices d'interaction entre des sujets, au milieu d'un projet commun, c'est-à-dire des modes de développement de la pensée en groupe.

C'est dans ce contexte que j'ai trouvé la nécessité du cinéaste Evaldo Mocarzel ${ }^{6}$ d'écrire des lettres/courriels pour les monteurs de ses documentaires. J'ai observé que ce moyen de communication était devenu nécessaire face à la difficulté de passer au monteur des questions de nature générale et sensible qui impliquaient une intense et longue expérience de registre avec les groupes.

Quant aux lettres comme documents d'interaction entre les membres du groupe, c'est le moyen rencontré de passer le relai sensible et conceptuel au monteur.

Un autre projet de théâtre, que j'ai accompagné, le projet Aisthesis : la création en flux (2015) offre un autre cas intéressant pour la discussion de documents de processus, générés par la nécessité du groupe, qui indique aussi le processus communicatif entre ses membres dans la constitution d'un projet commun. C'était un groupe formé de six artistes, membres de groupes différents qui se réunissaient pour une proposition de travail en commun.

Ils vivaient dans des villes différentes et se rencontraient périodiquement. Tout au long du processus, des enregistrements audiovisuels, photographiques, des expérimentations proposées ont été faits et il y a eu un échange intense de messages, à travers l'application WhatsApp, dans le but de rester en contact, de proposer des idées, de discuter de lectures, etc.

77 Il est intéressant d'observer que, dans le contexte de l'expérimentation contemporaine, cette forme de communication est revenue plus tard au moment de rendre public ce processus sous la forme de lecture dramatique d'une édition du long échange de messages.

Les cas présentés ici ne prétendent pas épuiser les possibilités infinies de documents générés selon les spécificités de chaque groupe, de chaque processus et des sujets impliqués.

79 En poursuivant les réflexions sur les spécificités d'un artiste déterminé et en dialogue avec l'expérimentation contemporaine, j'apporte une œuvre de Jérôme Bel'7. 


\section{Critique de processus et l'expérimentation contemporaine}

80 je vais discuter de la conférence-spectacle Mix, de Jérôme Bel (Itaú Cultural/São Paulo/ Brésil 2005).

Selon Bel, son œuvre Le dernier spectacle, qui s'appropriait les parties de chorégraphies d'autres chorégraphes, a été un succès critique, a rendu des thèses, mais n'a jamais reçu un bon accueil du public. Une œuvre qui médite sur des questions polémiques comme l'auctorialité, le copyright et les fausses identités. Quand il a été invité à la représenter, il a opté pour la reproduire sous ce nouveau format pour être mieux compris du public. Ces intentions, dans le cas de Bel, ne peuvent être comprises sans l'ironie et l'humour que son spectacle exhale.

82 Sans la possibilité de définir avec précision ce à quoi nous étions en train d'assister, ce que nous voyions sur la scène de théâtre était un artiste, assis à une table, donnant une conférence, à partir de consultations permanentes sur un ordinateur portable. Nous avons, ainsi, pris connaissance de ses dialogues, ses recherches, ses hésitations et ses certitudes. Il y avait encore une traductrice, faisant ses interventions à chaque pause du conférencier. Nous voyions aussi des extraits de la vidéo du spectacle, projetés à la demande de l'artiste. Il y avait aussi ses performances ou ses démonstrations, de manière à détailler certaines explications.

Comme nous pouvons l'observer, la discussion sur le copyright explicite l'auctorialité en réseau, dans la mesure où elle montrait les appropriations en vidéos et faisait ressortir les recherches réalisées sur le copyright, dans le domaine du droit. En même temps, nous assistions au transfert des archives de création sur la scène, comme part de la mise en scène.

84 Comme cette « conférence » faisait partie du spectacle Mix, elle était parfois interrompue, à des moments apparemment prédéfinis, par la performance Shirtology.

85 Mix n'a pas de délimitation du médium dans lequel l'œuvre se montre au public, ce qui pourrait être considéré comme l'une des traces des exploitations artistiques de la contemporanéité. Cette question est déjà mise en évidence par la dénomination conférence-spectacle, par la nécessité de la critique de faire face à l'indéfinition de frontières, en ce qui concerne les médias, les genres et, dans ce cas, spécialement, les expressions artistiques. Que regardons-nous? Une conférence? Un spectacle de théâtre sur la danse? Une mise en scène?

En pensant à une autre caractéristique de l'expérimentation artistique contemporaine, cette œuvre nous renvoie à une autre forme d'absence de frontières, en ce qui concerne la relation entre processus de production et l'œuvre montrée publiquement. De nombreux artistes, comme Jérôme Bel, offrent une grande diversité d'exploitations de ces limites.

Dans ce cas spécifique, le processus de création est transformé en œuvre. Au final, nous sortons avec l'impression que nous assistons, entre autres choses, au récit du processus de création de son «mal compris » Le dernier spectacle.

88 Immergés dans cette effervescence de l'expérimentation artistique qui dilue les frontières, nous arrivons à la fin de cet article qui a commencé par la présentation 
d'aspects généraux des processus de création, en passant par certaines spécificités du théatre et en arrivant aux singularités de certaines propositions.

Ce parcours a été réalisé à partir du regard de la critique de processus, qui se propose comme une approche critique, soutenue par la théorie de création dans le cadre de la complexité. Son expansion est alimentée par les défis de la contemporanéité.

\section{BIBLIOGRAPHIE}

ARAUjo, Antônio (2011). A gênese da Vertigem : o processo de criação de 'O paraíso perdido'. São Paulo : Editora Perspectiva.

BEL, Jérôme (2005). Mix. São Paulo : Itaú Cultural.

COLAPIETRO, Vincent (1989). Peirce's approach to the self: a semiotic perspective on human subjectivity. New York: State University of New York.

COLAPIETRO, Vincent (2003). « The loci of creativity: fissured selves, interwoven practices », Manuscrítica - Revista de crítica genética, 11. São Paulo : Annablume, pp. 59-82

DE MASI, Domenico (2005). Criatividade e grupos criativos : Fantasia e concretude. vol. 2. Rio de Janeiro : Sextante.

DE MASI, Domenico (org.) (2007). A emoção e a regra : os grupos criativos na Europa de 1850 a 1950. 9ª ed. Rio de janeiro : José Olympio.

EISENSTEIN, Serguei (1987). Memórias imorais. São Paulo : Companhia das Letras.

FELLINI, Federico (2015). Ensaio de uma orquestra. Projeto Aisthesis : a criação em fluxo. São Paulo : Itaú Cultural.

KANDINSKY, Wassily (1990). Do espiritual na arte. São Paulo : Martins Fontes.

LouIs, Murray (1992). Dentro da dança. Rio de Janeiro : Nova Fronteira.

MORIN, Edgar (1998). 0 método 4 : as ideias. Habitat, vida, costumes, organização. Porto Alegre : Sulinas. MORIN, Edgar (2000). A inteligência da complexidade. São Paulo : Peirópolis.

MUSso, Pierre (2004). « A filosofia da rede » in André Parente (org). Tramas da rede. Porto Alegre : Sulina.

PEIRCE, C. S. The collected papers of Charles Sanders Peirce. 8 vol. USA : Harvard Press.

\section{NOTES}

1. Conférence dans le Programme de $3^{\mathrm{e}}$ cycle en Communication et Sémiotique de l'Université Pontificale Catholique de São Paulo (PUC/SP) en 2014.

2. Voir l'ebook Salles, Cecilia A. Unfinished gesture: process of artistic creation https:// www.estacaoletras.com.br/product-page/unfinished-gesture-process-of-artistic-creation

3. Je me réfère aux trois catégories de la phénoménologie de Charles $\mathrm{S}$. Peirce. 
4. Façon de présenter des citations de l'œuvre Collected Papers de Charles S. Peirce, qui se réfère au volume 1, paragraphe, 205.

http://www.helsinki.fi/science/commens/dictionary.html

5. Directeur du théâtre du Vertige (São Paulo/Brésil), groupe de grande importance dans le théâtre contemporain avec un grand nombre de représentations au Brésil et à l'étranger. Vainqueur du prix Golden Medal du meilleur spectacle pour la pièce BR-3 à la Quadriennale de Prague, 2011.

https://www.teatrodavertigem.com.br/

6. Cinéaste brésilien qui a fait un projet avec 15 troupes de théâtre de São Paulo/Brésil. Il a accompagné et enregistré les processus qui ont engendré des documentaires.

7. Chorégraphe et danseur né à Montpellier.

\section{RÉSUMÉS}

L'objectif de cet article est de présenter la recherche sur les processus de création développée dans l'Université Pontificale Catholique de São Paulo/Brésil (PUC/SP) depuis 1990. Tout d'abord, nous ferons un bref historique de cette ligne de recherche, en discutant certains procédés méthodologiques. Ensuite, nous présenterons une proposition théorique pour une approche critique du processus, en pensant à plusieurs spécificités des parcours de création au théâtre. Il s'agit d'une possible théorie de la création artistique de base sémiotique (Peirce), en dialogue avec le concept de réseau (Musso), qui a engendré le concept de création comme un réseau en construction. C'est dans ce contexte que l'on propose une critique de processus. Finalement, nous discuterons de l'importance de cette approche pour des réflexions critiques sur l'expérimentation contemporaine, à partir d'une œuvre de l'artiste français Jérôme Bel.

The object of this article is to present the research on creative processes developed at the Pontifical Catholic University of São Paulo/Brazil (PUC/SP) since 1990. Primarily, we will make a brief history of this line of research, discussing some methodological procedures, followed by a theoretical proposal for a critical approach to the process, considering some specificities of the creative paths of the theatre. It is a theory of creative processes of semiotic basis (Peirce), in dialogue with the concept of network (Musso), which led to the concept of creation as a network under construction. that supports what I have been calling criticism of creative processes. Finally, we will discuss the relevance of this approach for critical reflections on contemporary experimentation, based on a work of the French artist Jérôme Bel.

\section{INDEX}

Keywords : Creative process, theatre, criticism, network, contemporary exerimentation

Mots-clés : Processus de création, théâtre, critique, réseau, expérimentation contemporaine 


\section{AUTEUR}

\section{CECILIA ALMEIDA SALLES}

Université Pontificale Catolique de São Paulo/Brésil

cecilia.salles[at]gmail.com 